\section{Case Reports in Ophthalmology}

\title{
Secondary Angle Closure due to Crystalline Lens Dislocation in a Patient with Atopic Dermatitis and Chronic Eye Rubbing
}

\author{
Justin Kuiper Mark Slabaugh \\ Department of Ophthalmology, William H. Havener Eye Institute, The Ohio State \\ University Medical Center, Columbus, $\mathrm{OH}$, USA
}

\section{Keywords}

Atopy $\cdot$ Angle closure $\cdot$ Eye rubbing $\cdot$ Glaucoma $\cdot$ Ectopia lentis

\begin{abstract}
Purpose: To report an unusual case of ectopia lentis and angle closure in a patient with chronic eye rubbing. Methods: A 57-year-old male with a history of poorly controlled atopic dermatitis presented with right eye pain, decreased vision, and an intraocular pressure (IOP) of $55 \mathrm{~mm} \mathrm{Hg}$. He had no past history of ocular disease and no reported history of trauma. He did report a history of chronic eye rubbing. Results: Best corrected visual acuity was hand motions. The examination revealed severe atopic keratoconjunctivitis in both eyes, microcys tic corneal edema of the right eye, and 2+ nuclear sclerosis in both eyes. Gonioscopy showed no visible angle structures OD and an open angle OS. Topical and oral IOP-lowering medications and a laser iridotomy were unsuccessful at lowering IOP. He was taken to the operating room for a lensectomy and was found to have 9 clock hours of zonular dehiscence and a dislocated lens. After lensectomy, the IOP improved to $9 \mathrm{~mm} \mathrm{Hg}$ on postoperative day 1. A follow-up examination at 2 weeks showed improved acuity to 20/150 with a pinhole and an IOP of $10 \mathrm{~mm} \mathrm{Hg}$. A dilated examination OS did not reveal significant phacodonesis, and the patient was referred for a possible sutured sulcus lens or anterior chamber intraocular lens. Conclusions: It is important for the provider to consider ectopia lentis in the differential for patients with pupillary block angle closure. For patients with atopic disease, one should be
\end{abstract}




\section{Case Reports in Ophthalmology}

aware that eye rubbing may be a cause of zonular dehiscence, even in the absence of reported trauma or prior intraocular surgery.

\section{Introduction}

Ectopia lentis is a known cause of lens-induced angle closure, which is particularly challenging to diagnose and manage [1]. Angle closure in these patients is due to zonular insufficiency, which allows the lens to subluxate or dislocate anteriorly, resulting in pupillary block. Frequent causes of zonular insufficiency include trauma, pseudoexfoliation, Marfan syndrome, and Ehlers-Danlos syndrome; the most common cause is trauma. Less frequently observed causes include homocystinuria, Weill-Marchesani syndrome, sulfite oxidase deficiency, retinitis pigmentosa, simple ectopia lentis, and ectopia lentis et pupillae [1]. Traumatic causes may be further subdivided into blunt trauma, penetrating trauma, iatrogenic trauma, and chronic repetitive trauma.

We present an unusual case of ectopia lentis from eye rubbing in a patient with atopic dermatitis, resulting in acute angle closure.

\section{Case Report}

A 57-year-old male with no past history of ocular disease presented as a transfer from an outside hospital for angle closure. Intraocular pressure (IOP) prior to transfer had been measured with a Tono-Pen (Reichert, Depew, NY, USA) to be $55 \mathrm{~mm} \mathrm{Hg}$. On arrival, he complained of severe headache, nausea, and blurry vision in his right eye. He noted that the symptoms had started while watching TV earlier in the day. He denied any history of antecedent trauma to either eye. His past medical history was significant for severe atopic dermatitis, which was poorly controlled even with the use of infused biologic therapy, as well as a history of asthma.

The examination revealed "hand motion" vision OD, with IOP measured by Goldmann applanation tonometry to be $48 \mathrm{~mm} \mathrm{Hg}$. An external examination revealed thickened, scalyappearing skin, and a slit-lamp examination showed thickened eyelid skin, severe conjunctival injection, a thickened conjunctiva, and a papillary reaction in both eyes (Fig. 1). The patient had microcystic corneal edema OD, and was found to have 2-3+ nuclear sclerotic cataracts in both eyes. Gonioscopy OD revealed no visible angle structures, while the OS was open to the anterior ciliary body $360^{\circ}$.

The patient was initiated on topical IOP-lowering medications and oral Diamox as well as hyperosmotic agents. A laser peripheral iridotomy was attempted without relief of the pupillary block, and the IOP remained at $51 \mathrm{~mm} \mathrm{Hg}$ after the procedure. After several hours of no response to medical or laser therapy, he was taken to the operating room for emergent surgery, which was planned to include phacoemulsification and possible surgical iridectomy or trabeculectomy. After the initial incisions had been constructed and the pupil dilated with a Malyugin ring, the anterior capsule was stained with trypan blue and a capsulorrhexis was initiated. It became immediately apparent that there were 9 clock hours of zonular dehiscence and a partially dislocated crystalline lens. The lens was removed completely with phacoemulsification, and a limited anterior vitrectomy was performed. The anterior chamber became deep, and no iridectomy or trabeculectomy was performed. 
On postoperative day 1, the patient's IOP was $9 \mathrm{~mm} \mathrm{Hg}$ by Goldmann applanation tonometry, and he had relief of the eye pain symptoms. The further history acquired at that time revealed that the patient had been rubbing his eyes aggressively on a daily basis due to severe itching of the eyes and eyelid skin. On the follow-up examination 2 weeks postoperatively, his vision was 20/150 with an aphakic correction, and the IOP was $10 \mathrm{~mm} \mathrm{Hg}$ on prednisolone and ofloxacin. A dilated examination OS did not reveal significant observable phacodonesis. Anterior segment ultrasound biomicroscopy and OCT were performed, which showed mild anterior lens vaulting in the left eye, as well as partial anterior iris vaulting and a deep chamber with aphakia in the right eye (Fig. 2). Prophylactic peripheral iridotomy in the left eye and a secondary sutured intraocular lens for the right eye were performed for further visual rehabilitation.

\section{Discussion}

Previous case reports have shown an association between eye rubbing and a number of eye problems, including keratoconus [2], cataracts [3], retinal tears [4], and uveitis [5]. There have also been a number of case reports of eye rubbing causing posterior chamber intraocular lens dislocation from zonular dehiscence [6] and capsule rupture [7]. We were able to find one case report of a patient whose eye rubbing resulted in crystalline lens dislocation [7]. To our knowledge, however, this is the first case report of a patient presenting with angle closure after crystalline lens dislocation due to eye rubbing.

Often, eye conditions associated with chronic eye rubbing occur in patients with atopic dermatitis. Atopic dermatitis (eczema) is an inflammatory skin condition; ocular manifestations have been reported to occur in $25-43 \%$ of patients with atopic dermatitis and may include keratoconjunctivitis, blepharitis, and eyelid dermatitis [8,9]. All of these findings were present in our patient (Fig. 1). Type I and type IV hypersensitivity reactions have been implicated as the cause of these symptoms, and in patients with atopic dermatitis and atopic keratoconjunctivitis, serum and tear IgE levels are elevated [10]. There is also an elevation of local mast cells and eosinophils, which can produce intense pruritus [9]. In patients with poorly controlled symptoms, constant itching and eye rubbing can become a source of repetitive trauma to the eye.

The proposed mechanism of zonular injury is that pressure in the anterior-posterior plane results in shortening of the axial length and subsequent expansion equatorially, which may stretch and damage the zonules. This can eventually lead to zonular weakness and dehiscence [11]. Although primarily of historical interest, the ability to manually rupture the zonules led to the development of the blunt couching technique for correction of blindness due to cataract. The outcomes of this technique are generally poor, although it is still performed in some underserved areas [12]. Of note, our patient was right-handed, and his lens dislocation was found to be in his right eye. This is consistent with previous studies of eye rubbing and keratoconus, which have suggested that eye pathology associated with eye rubbing is more likely to occur on the side of the patient's dominant hand [13].

The management of this patient was the same as for others with traumatic lens dislocation and secondary angle closure. Initial treatment of an elevated IOP may include IOPlowering medications. To relieve pupillary block, a laser peripheral iridotomy may be performed. In the setting of suspected ectopia lentis and secondary angle closure, one should consider performing two laser peripheral iridotomies $180^{\circ}$ apart [14]. Ultimately, however, 
removal of the lens may be required to relieve the pupillary block, as was demonstrated in our patient.

Cataract extraction in these patients may be complicated due to lack of zonular support, and the surgeon should consider the use of capsule hooks, capsular tension rings, or capsular tension segments to stabilize the capsule [15]. Depending on the stability of the capsule, the patient may require an anterior chamber intraocular lens or sutured sulcus lens [15].

This case report is a unique presentation of secondary angle closure due to zonular dehiscence and lens dislocation from eye rubbing. It is important for the provider to consider ectopia lentis in the differential for patients with pupillary block angle closure. For patients with atopic disease, one should be aware that eye rubbing may be a cause of zonular dehiscence, even in the absence of reported trauma or prior intraocular surgery.

\section{Statement of Ethics}

Consent was obtained from the patient to present this case report.

\section{Disclosure Statement}

The authors have no conflicts of interest to disclose.

\section{References}

1 American Academy of Ophthalmology: Lens and Cataract. 2016-2017 BCSC Basic and Clinical Science Course, vol 11, pp 39-43.

2 McMonnies CW: Abnormal rubbing and keratectasia. Eye Contact Lens 2007;33(pt 1):265-271.

3 Nagaki Y, Hayasaka S, Kadoi C: Cataract progression in patients with atopic dermatitis. J Cataract Refract Surg 1999;25:96-99.

4 Kothari N, Young RC, Read SP, Tutiven J, Perez VL, Flynn HW Jr, Berrocal AM: Retinal detachment associated with atopic dermatitis. Ophthalmic Surg Lasers Imaging Retina 2017;48:513-517.

$5 \quad$ Singh G, Mathur JS: Atopic erythroderma with bilateral cataract, unilateral keratoconus and iridocyclitis, and undescended testes. Br J Ophthalmol 1968;52:61-63.

6 Yamazaki S, Nakamura K, Kurosaka D: Intraocular lens subluxation in a patient with facial atopic dermatitis. J Cataract Refract Surg 2001;27:337-338.

7 Peter NM, Nath R, Tranos PG, Teimory M: Bilateral lens subluxation associated with atopic eczema. Eur J Ophthalmol 2005;15:409-411.

8 Bielory B, Bielory L: Atopic dermatitis and keratoconjunctivitis. Immunol Allergy Clin North Am 2010;30:323-336.

9 Chen JJ, Applebaum DS, Sun GS, Pflugfelder SC: Atopic keratoconjunctivitis: a review. J Am Acad Dermatol 2014;70:569-575.

10 Eiseman AS: The ocular manifestations of atopic dermatitis and rosacea. Curr Allergy Asthma Rep 2006;6:292-298.

11 Marcus DM, Topping TM, Frederick AR Jr: Vitreoretinal management of traumatic dislocation of the crystalline lens. Int Ophthalmol Clin 1995;35:139-150.

12 Isawumi MA, Kolawole OU, Hassan MB: Couching techniques for cataract treatment in Osogbo, South West Nigeria. Ghana Med J 2013;47:64-69.

13 Rabinowitz YS, Nesburn AB, McDonnell PJ: Videokeratography of the fellow eye in unilateral keratoconus. Ophthalmology 1993;100:181-186.

14 American Academy of Ophthalmology: Glaucoma. 2016-2017 BCSC Basic and Clinical Science Course, vol 10, pp 129-130.

15 Hoffman R, Snyder ME, Devgan U, Allen QB, Yeoh R, Braga-Mele R; ASCRS Cataract Clinical Committee; Challenging/Complicated Cataract Surgery Subcommittee: Management of the subluxated crystalline lens. J Cataract Refract Surg 2013;39:1904-1915. 


\section{Case Reports in Ophthalmology}
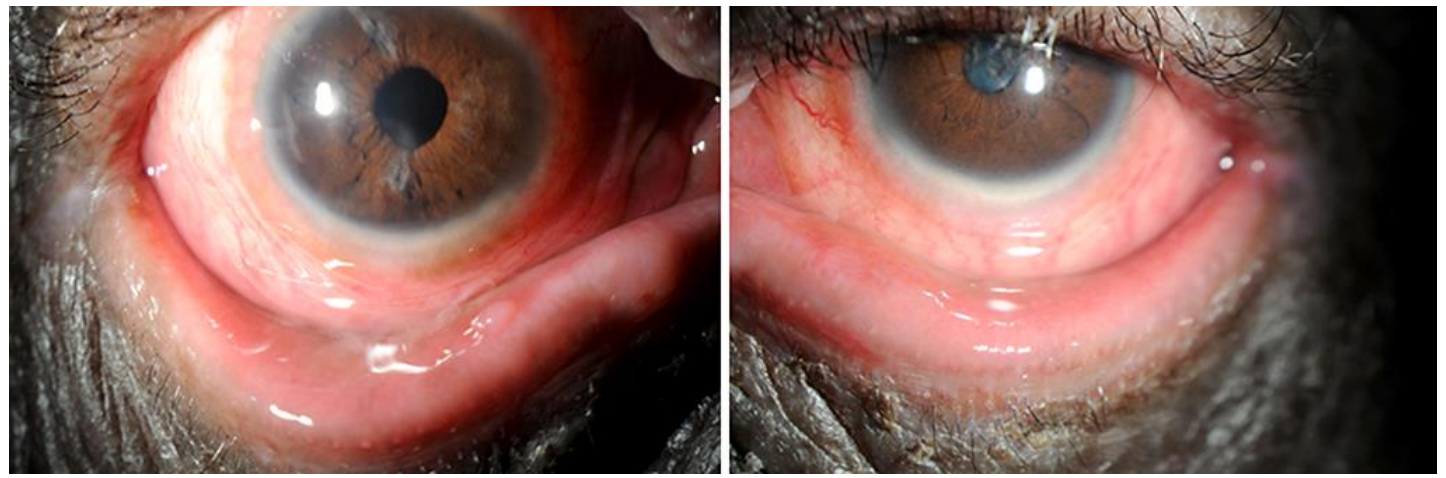

Fig. 1. Slit lamp photographs demonstrating eyelid thickening and conjunctival injection and thickening consistent with severe atopic keratoconjunctivitis OD (right) and OS (left). Note: the lower lid was retracted for exposure of the palpebral conjunctiva.
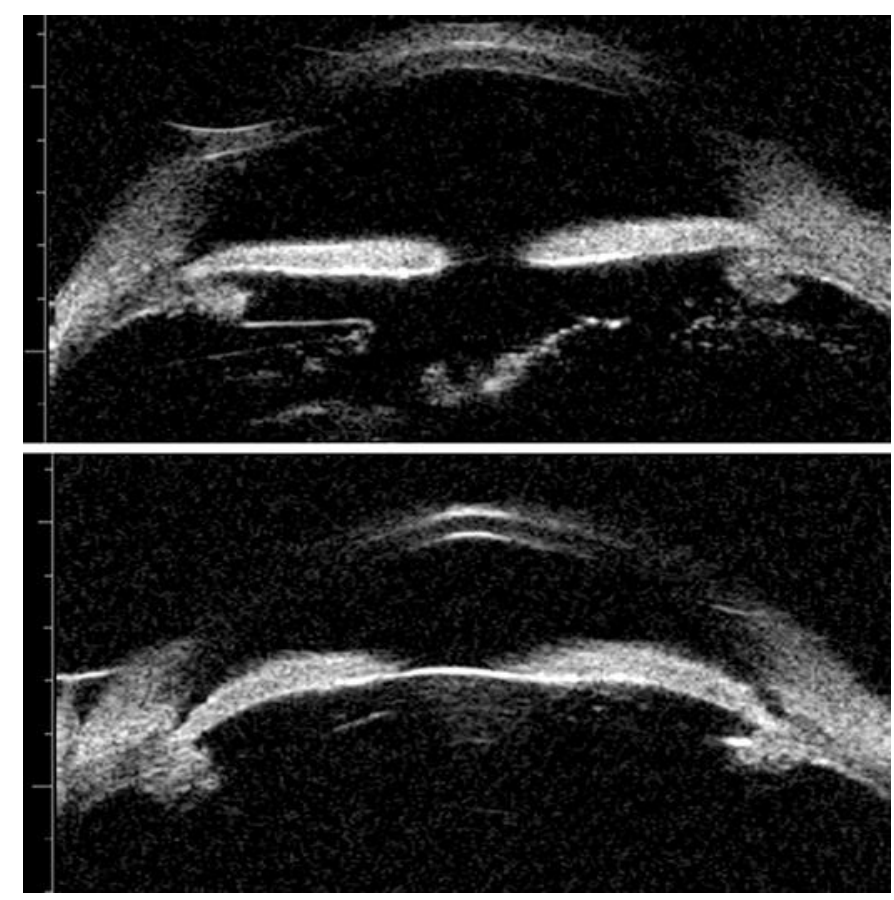

Fig. 2. Postoperative anterior segment ultrasound revealing iris flattening and aphakia OD (top) and a stable crystalline lens with anterior vaulting and an open angle OS (bottom). 